Lucrările Seminarului Geografic Dimitrie Cantemir

Vol. 44, October 2017, pp. 43-68

http://dx.doi.org/10.15551/lsgdc.v44i0.04

\title{
Innovation et développement territorial durable : gouvernance et outils open source pour deux projets d'ecoquartiers
}

\section{Anthony Tchékémian ${ }^{1}$}

${ }^{1}$ Université de la Polynésie Française, Tahiti - Polynésie française

To cite this article: Tchékémian, A. (2017). Innovation et développement territorial durable : gouvernance et outils open source pour deux projets d'ecoquartiers. Lucrările Seminarului Geografic Dimitrie Cantemir, Vol. 44, pp. 43-68. DOI: 10.15551/lsgdc.v44i0.04

To link to this article: http://dx.doi.org/10.15551/lsgdc.v44i0.04 


\title{
INNOVATION ET DÉVELOPPEMENT TERRITORIAL DURABLE : GOUVERNANCE ET OUTILS OPEN SOURCE POUR DEUX PROJETS D'ECOQUARTIERS
}

\author{
Anthony Tchékémian ${ }^{1}$
}

Résumé: Quels outils du Web 2.0 permettent aux acteurs des territoires 2.0 la mise en place d'une bonne gouvernance ? Cette gouvernance se révèle-t-elle particulièrement adaptée dans le cadre d'un aménagement du territoire basé sur l'innovation et le développement durable ? L'intérêt de cette recherche-action repose sur les attentes et demandes en matière de gouvernance, par l'étude de deux projets d'écoquartiers : l'un, lorsqu'il s'agit de la volonté de citoyens regroupés et organisés en autopromotion ; l'autre, lorsque la décision provient d'une collectivité territoriale. En mobilisant l'approche Living Lab, des outils open source accessibles depuis le Web 2.0 sont proposés aux acteurs en vue de la mise en place d'une bonne gouvernance. L'étude atteste de la pertinence de l'échelle locale en matière d'aménagement durable des territoires et de la pertinence d'outils open source pour la mise en place d'une bonne gouvernance.

Mots clés : web 2.0 - territoire 2.0 - open source - gouvernance - living lab - écoquartiers

\section{Introduction : du Web 2.0 au territoire 2.0}

Les décisions d'aménagement, ne serait-ce qu'à un échelon très localisé, se prennent souvent dans une logique d'approche descendante, ce qui les rend souvent éloignées des réalités de terrain et besoins quotidiens des acteurs (Lacour, Delamarre, 2010 : 168) ${ }^{2}$. De plus, le cloisonnement des différents acteurs (publics, privés, associatifs, usagers) et le manque de pluridisciplinarité au sein des équipes de conception, réalisation et suivi des projets, ne permettent pas de répondre correctement aux attentes de plus en plus précises des usagers, trop peu consultés (Farinos, 2009 : 89).

\footnotetext{
${ }^{1}$ Maître de conférences en géographie et aménagement du territoire, Université de la Polynésie Française UMR 241 EIO, B.P. 6570 - 98702 Faa'a, Tahiti - Polynésie française, anthony.tchekemian@upf.pf

${ }^{2}$ «L'efficacité insuffisante de l'aménagement a été et est encore inhérente à une gouvernance insuffisante ; des fragilités croissantes, d'essence exogène, sont à observer, même dans le cadre européen » (Lacour, Delamarre, 2010 : 168). Ainsi, même s'il demeure quelques incohérences, une bonne gouvernance s'avère un facteur crucial dans la réussite d'un projet d'aménagement. Cependant, inclure une participation en vue d'une bonne gouvernance requiert de profonds changements dans la façon d'appréhender un projet collectif. L'efficacité insuffisante de l'aménagement est due à une mauvaise gouvernance, il faut donc, à partir des recommandations de ces auteurs, s'essayer à optimiser les méthodes actuelles, voir à en expérimenter de nouvelles.
} 
Pourtant, à l'ère du Web $2.0^{3}$, qui évolue vers plus de simplicité et d'interactivité, les échanges et outils se sont spontanément développés. L'avènement des smartphones, tablettes, ordinateurs portables permettent de localiser, partager les informations et expériences, quant à la proximité et qualité d'un lieu et service (musée, restaurant, parking, hôpital...), que les usagers sont en droit d'attendre, en temps réel, dans les territoires vécus et partagés. L'émergence de ces véritables réseaux participatifs n'a qu'une existence virtuelle, mais elle influe sur le monde réel dans les choix, les décisions des usagers, en leur transmettant un certain nombre d'informations (via des sites comme : DismoiOù ${ }^{4}$, Cityvox ${ }^{5}$ ). Toutes ces initiatives et ces outils attestent que de simples usagers ne comptent plus uniquement sur les pouvoirs publics pour s'organiser et s'informer, mais en créent de nouveau. D'autant plus que ces informations arrivent et se transmettent en temps réel, ce qui augmente leur efficacité.

Des initiatives sont déjà engagées par des collectivités territoriales, comme au travers du label national « Ville Internet » qui depuis 1999, récompense les collectivités françaises qui inscrivent une politique numérique dans leur mission de service public ${ }^{6}$. L'annuaire géolocalisé Comarquage. $\mathrm{fr}^{7}$ rassemble, via l'outil Google Earth $^{8}$, des services de proximité, des informations sur la santé, l'éducation, l'administration, l'emploi, la vie pratique, le sport et la culture. Ce fonctionnement en plateforme de réseau et de partage des informations en temps réel peut être étendu à d'autres services urbains comme la mobilité (disponibilité des vélos en libre-service, infos sur le réseau des transports en commun, le trafic routier, permanences des services de santé...), à la qualité de vie grâce à la présence de capteurs et collecteurs de données urbaines (caméras de surveillance, capteurs de qualité de l'air...). Ou encore, le programme d'action ville 2.0, initié en 2007 par la Fondation Internet Nouvelle Génération (FING) qui tente d'inventer et d'expérimenter la ville de demain, en incluant l'usager, afin de faire face aux changements rapides des problématiques urbaines (mobilité,

\footnotetext{
${ }^{3}$ L'expression Web 2.0 est utilisée pour la première fois, en 2004, par Dale Dougherty, puis popularisée par Tim O'Reilly. La définition du web 2.0 s'imposera à partir de 2007, " comme étant la conception de systèmes qui mettent à profit les effets des réseaux sociaux pour tirer le meilleur de ceux qui les utilisent, ou pour parler plus simplement, mettre à profit "l'intelligence collective" » (Duperrin, 2008). Le Web 2.0 désigne donc une évolution de l'outil internet, en tant que plateforme. Le web propose des applications qui n'ont plus besoin d'être installées et qui sont exécutables depuis un navigateur. Ainsi, utilisateur du Web 2.0 devient acteur : il peut partager ses fichiers, travailler en collaboration, enrichir la plateforme Internet... Voir Boussard (2011).

${ }^{4}$ Dismoioù est un service collaboratif de recommandations de bonnes adresses. Il s'agit d'une application française de géolocalisation [URL : http://dismoiou.fr/].

${ }_{6}^{5}$ Cityvox est un site français de critiques de restaurants.

${ }^{6}$ Voir site [http://www.villes-internet.net], consulté le 15 mai 2014, qui, ce même jour, recense 14459 initiatives locales d'usage ou de services numériques par les élus et les agents des collectivités territoriales.

${ }^{7}$ L'annuaire de l'Administration Comarquage.fr contient actuellement près de 70000 fiches d'organismes et de services géolocalisés sur le territoire français, voir site, [URL : http://www.comarquage.fr/Annuaire-deproximite], consulté le 15 mai 2014.

${ }^{8}$ Google Earth est un logiciel développé par Google, permettant de parcourir la Terre par des images satellite, en 2D ou en 3D. il s'agit donc d'un logiciel de visualisation d'images satellites et photos aériennes compilées, chargées en mode streaming, en provenance des serveurs de Google. Il est également possible d'effectuer des recherches (restaurants, hôtels, etc.) et de consulter des itinéraires routiers. Voir site [URL : https://www.google.fr/intl/fr/earth/], consulté le 26 juillet 2016.
} 
innovation, compétitivité, durabilité, cohésion sociale, vieillissement...), les technologies y sont employées à la fois comme instrument et catalyseur? .

Le concept de ville 2.0 est dérivé de celui de Web 2.0. Dans le cas du web, elle désigne les technologies et les usages d'une nouvelle génération qui permettent aux internautes sans compétences particulières en informatique de s'approprier les fonctionnalités du web (réseaux sociaux, sites collaboratifs...). Il devient ainsi possible aux usagers de la toile, non seulement d'échanger des informations, mais également d'interagir de façon simple avec le contenu et la structure des pages (qui dans sa version première génération, était plutôt austère pour les non-initiés...). Le Web 2.0 marque aussi l'avènement de toute une génération de réseaux sociaux et de plateformes collaboratives qui peuvent s'avérer des outils pertinents pour l'innovation territoriale et la gouvernance.

D'une manière générale, il s'agit de développer de plus en plus d'applications technologiques appliquées au territoire. Pour David Fayon (2010) le Web 2.0 marque une évolution des services qui sont constitués par les données collectées et stockées. C'est-à-dire qu'il marque une évolution vers une participation accrue de l'usager, de nouveaux usages et des citoyens mieux informés et plus exigeants (comparaison des prix, des caractéristiques...). Ainsi, l'internaute du Web 2.0 devient « consommacteur » car il peut se baser sur les avis d'autres consommateurs pour choisir un produit ou un service, et il devient « consommauteur » car il peut lui-même devenir l'auteur d'informations qui vont nourrir la masse d'informations disponible pour d'autres usagers (Fayon, 2010). Ces évolutions induisent des changements profonds au sein de notre société : accessibilité des données en permanence et quel que soit le lieu grâce aux smartphones, la généralisation du e-achat, la « googlelisation ${ }^{10} \gg$ des esprits, un savoir plus consensuel (tel qu'un Wiki ${ }^{11}$ ) et une meilleure information.

Aujourd'hui, les territoires, les villes sont de plus en plus représentées, analysées, photographiées, commentées et cartographiées sous tous les angles (Google Earth, Geoportail, Openstreetmap...). Le Web 2.0 bouleverse les pratiques et facilite grandement le partage et la géolocalisation des informations. Toujours plus vite, l'information est accessible partout et de manière plus précise, le Web 2.0 tend donc naturellement à devenir un réseau de systèmes d'informations géographiques pour la plus part ouverts et contributifs. Ainsi l'usager, émancipé, a pris pour habitude d'être le propre acteur de son territoire virtuel. Si l'on étend cette notion à celle d'un territoire réel, d'une ville plus précisément, on se rend compte qu'il existe des analogies. En effet, dans une société largement ouverte et structurée autour des Technologies de l'Information et de la Communication (TIC), où l'information est clairement la nouvelle ressource stratégique et où l'innovation fait désormais partie du

\footnotetext{
${ }^{9}$ En deux ans, «Villes 2.0 » a réuni plus de 1500 personnes lors de 3 manifestations publiques, deux Carrefours des possibles, plus de 25 ateliers et conférences et de 30 interventions publiques à Paris, Bordeaux, Brest, Lille, Lyon, Marseille, Nantes, Nice, Rennes, Toulon, Toulouse... ainsi qu'Amsterdam, Bruxelles, Genève et Stuttgart. Villes 2.0 a attiré l'attention des médias, des élus, des entreprises des services urbains et de réseaux de professionnels, consultants, urbanistes, architectes. Plusieurs projets d'expérimentation en phase d'élaboration se sont concrétisés en 2009. Voir lien [URL : http://fing.org/?Bilan-du-programme-Villes-2-0-en], consulté le 15 mai 2014.

${ }^{10}$ Selon Fayon (2010) la « googlelisation » est le réflexe d'aller chercher sur Google la réponse à une question que l'on se pose.

${ }^{11}$ Selon le Dictionnaire de l'informatique et d'Internet, un Wiki est un site web dynamique dont tout visiteur peut modifier les pages à volonté. Il permet donc de communiquer ses idées rapidement. Voir [URL : http://www.dicofr.com/cgi-bin/n.pl/dicofr/definition/20040313125252], consulté le 12 juillet 2013.
} 
mode de vie, les citoyens résidents d'une agglomération, pourtant sans compétence particulière en urbanisme, en aménagement ou en architecture, font ressentir de plus en plus fortement aux élus et aux acteurs de l'aménagement qu'ils souhaitent devenir des acteurs à part entière.

En matière d'aménagement urbain, notamment dans la conception de projets, les usagers, qui vivent et animent leur territoire au quotidien, sont les mieux placés pour proposer et faire émerger les différents besoins et attentes auxquelles les décideurs doivent répondre. Chaque concepteur, aussi doué soit-il, a besoin de questionner les utilisateurs, afin d'élaborer de nouveaux produits et services, en fonction de l'évolution des besoins de chacun. Les acteurs se doivent d'innover en permanence pour être compétitifs (Kaplan, 2009 : 104) et pour que les territoires 2.0 (urbains, périurbains, ruraux) soient toujours plus attractifs $^{12}$. Ainsi, la mise en place d'outils numériques, libres et gratuits accessibles aux usagers du territoire peut permettre d'œuvrer à gouvernance la plus adaptée et optimale.

Selon l'ITGI (Information Technology Governance) ${ }^{13}$, la gouvernance a « pour but de fournir l'orientation stratégique, de s'assurer que les objectifs sont atteints, que les risques sont gérés comme il faut et que les ressources sont utilisées dans un esprit responsable ». Elle veille en priorité au respect des intérêts des « ayants droits » (citoyens, pouvoirs publics, partenaires, actionnaires...) et à faire en sorte que leurs voix soient entendues dans la conduite des affaires ${ }^{14}$. Issu de la théorie micro-économique et de la science administrative anglo-saxonne, la notion de «bonne gouvernance » a été diffusée dans les années 1990 par la Banque mondiale, comme la condition nécessaire des politiques de développement. La gouvernance repose sur quatre principes fondamentaux : la responsabilité, la transparence, l'Etat de droit, la participation.

$\mathrm{Si}$, en France, la décentralisation a permis de rapprocher l'État du terrain, permettant de ce fait à des organes locaux d'administrer eux-mêmes leurs territoires respectifs, le citoyen demeure le grand oublié du processus (Calame, 2008). Tout l'enjeu de la gouvernance est de réussir à inclure ce dernier dans la réflexion, la conception, l'organisation et l'aménagement du territoire, en tant qu'acteur. En effet, si l'Etat demeure le garant d'un cadre légal, le citoyen, bénéficiaire et utilisateur final, peut aussi en être, à la base, un des moteurs créateurs d'innovation. Cela implique inévitablement une refonte des pratiques et des modes de pensée actuels. Il faudra alors ouvrir un dialogue avec l'usager et les différents acteurs, plus souple et plus sensible aux aspects socioculturels, qui s'appuieraient sur une volonté politique affichée d'œuvrer pour le bien commun. Cette volonté ne peut se traduire qu'en valorisant le savoir et les savoir-faire de tous les acteurs de la communauté. Ainsi, la gouvernance est l'aptitude à développer des synergies fondées sur le développement de stratégies concertées entre l'état, les villes, les diverses collectivités territoriales et les acteurs de la société civile (Wackermann, 2005).

L'innovation territoriale est un concept qui peut sembler découler en partie de la notion de territoire 2.0. Selon Giraud (2009) la géographie dans son intérêt et son

\footnotetext{
${ }^{12}$ A ce propos, « réseaux et territoires sont dans le même rapport : l'espace géographique, qui les inclut tous les deux, est Janus à double face, aréal et réticulaire ; c'est le capillaire qui unit et lève la contradiction; et c'est pourquoi l'aménagement du territoire devrait le soigner particulièrement au lieu de se limiter aux « gros tuyaux », ceux des infrastructures lourdes » (Brunet, $2005: 481$ ).

${ }^{13}$ ITGI ou IT Governance se traduit en français par l'Institut de gouvernance de la technologie de l'information.

${ }^{14} \mathrm{D}$ 'après le lexique de L'organisation administrative du parlement, [URL : http://formationparlementaire.org/index.php/lexique], consulté le 14 juillet 2014.
} 
enthousiasme pour l'innovation à tendance à multiplier les définitions selon que l'angle d'approche soit social, technologique, culturel, institutionnel et territorial. Quelle que soit la définition qu'on lui choisit l'innovation territoriale peut avoir un lien direct avec les notions de Web 2.0, de territoire 2.0 et de gouvernance, c'est le cas de l'organisation en réseau, de la démarche participative et collaborative, ainsi que l'usage d'applications des outils numériques, etc. D'après Gerbaux et Giraud (2000 : 13-15), l'innovation territoriale doit dorénavant «se déployer sur les modalités d'articulation, d'association, de complémentarité et de régulation politique des dynamiques territoriales ». A ce titre, les deux projets d'écoquartier ${ }^{15}$ abordés dans cette recherche constituent une démarche d'innovation territoriale (technique, technologique, conceptuelle, gouvernance).

Le concept de Living Lab est développé à la fin des années quatre-vingt-dix par William J. Mitchell, chercheur au Massachusetts Institute of Technology (MIT). Son approche repose sur la création, à l'échelle d'un territoire, des ressources nécessaires pour accélérer les processus d'innovation et leur mise sur le marché, tout en réduisant les risques inhérents à ce genre d'expérimentations. La méthode consiste à organiser un espace d'informations, d'échanges et de rencontres entre l'ensemble des partenaires d'un projet : chercheurs, entreprises publiques et privées, collectivités, société civile... Mitchell justifie cette approche par la façon dont les villes ont évolué ces dernières décennies et la manière de les aborder aujourd'hui. Il développe le fait que les territoires sont digitalisés, impliquant une nouvelle gouvernance et la nécessité d'élaborer une nouvelle cartographie. Puis, en 2005, Europe par Nokia, qui développe un partenariat avec les usagers (Lixon, 2012), avant de devenir, en 2006, un programme européen organisé en réseau, nommé European Network of Living Labs (ENoLL) ${ }^{16}$.

Pour l'Union européenne, le Living Lab est un outil permettant de rendre les processus d'innovation plus efficaces en comblant le fossé entre la recherche et le marché ${ }^{17}$. Grâce à des partenariats entre les citoyens, les entreprises et les pouvoirs publics, la démarche Living Lab permet aux usagers et aux industries de tester les meilleures innovations pour demain dans les domaines de l'information et de la communication. En faisant le lien avec la définition de ville 2.0 et d'innovation territoriale, on peut apercevoir des pistes de réflexion afin d'élargir le concept de Living Lab à la recherche en aménagement du territoire. Les écosystèmes d'innovation ouverte dans la vie réelle constituent une nouvelle forme de pensée axée sur l'utilisateur, dans lequel ce dernier est entièrement intégré dans le processus de cocréation des nouveaux services, produits et infrastructures sociétales. En incluant

\footnotetext{
${ }^{15}$ Un écoquartier est un concept en lien avec le développement durable s'inscrivant dans une logique de renouveau destiné à amorcer la mutation du tissu urbain en tissu écologique, agréable, novateur et responsable. Selon Charlot-Valdieu, Outrequin (2009) il s'agit d' " un quartier conçu (ou renouvelé) avec une démarche environnementale, laquelle porte notamment sur le paysage ou la "végétalisation des quartiers" et la qualité environnementale des bâtiments (le plus souvent encore aujourd'hui des bâtiments neufs) ». Ce concept rassemble en une même vision idéalisée de la ville future toutes les améliorations qu'il est possible d'imaginer, aujourd'hui, pour réaliser les conditions du bien-être en ville, là où $80 \%$ de la population du nouveau millénaire devra vivre (Lefèvre, Sabard 2009). Ce qui implique donc une gouvernance efficace et fédératrice. A ce titre, l'écoquartier constitue donc une démarche d'innovation territoriale prometteuse (innovation technique et technologique, innovation conceptuelle, innovation dans la gouvernance).

${ }^{16}$ Voir site [URL : http://www.openlivinglabs.eu/], consulté le 14 juillet 2016.

${ }^{17}$ Voir site Europa, Information Society and Media, «Living Labs for user driven open innovation », [URL : http://ec.europa.eu/information_society/activities/livinglabs/index_en.htm], consulté le 14 avril 2011.
} 
l'utilisateur final dans le processus de création dès les étapes de développement, les besoins des utilisateurs sont ainsi mieux écoutés et respectés. Aussi, dans cette étude, l'approche Living Lab se définit comme une méthode de recherche en innovation ouverte qui vise le développement d'outils issus du Web 2.0 en vue d'une bonne gouvernance. Cette approche repose sur un processus de cocréation entre décideurs et usagers, au sein de deux projets d'écoquartiers, entre l'ensemble des acteurs (institutions, entreprises, habitants) dans des conditions réelles et s'appuie sur un écosystème de partenariats public-privé-citoyen.

En effet, aujourd'hui, les usagers souhaitent faire partie intégrante des processus de réflexion, conception et réalisation en lien direct avec leur vie quotidienne. Plus que d'en faire simplement ressentir le besoin, on peut également constater que certains n'ont pas attendu pour s'organiser spontanément, à la manière d'un réseau social, pour échanger, discuter, voir réaliser des projets d'aménagement de leur territoire. Ainsi, un Living Lab est un réseau d'acteurs pluridisciplinaires qui se regroupent dans le but d'innover et d'inventer les nouveaux produits et services de demain en se basant sur un ensemble d'applications permettant d'optimiser le travail en commun, souvent issues du Web 2.0, le tout, structuré par une bonne gouvernance. Peu importe la démarche envisagée pour vu que l'utilisateur final se retrouve au cœur du processus. De ce fait, l'approche Living Lab permet d'apporter quelques réponses et exemples concrets de ce qu'un processus d'innovation sociale structuré autour d'une bonne gouvernance et soutenu par les nouvelles technologies 2.0 peut apporter aux territoires et ainsi, entrer dans le cadre de l'innovation territoriale et de l'aménagement urbain.

En s'appuyant sur deux exemples d'écoquartiers ${ }^{18}$ et en essayant de définir une méthode concrète en vue de la mise en place de Living Labs appliqués aux nouveaux enjeux urbains et sociaux, la problématique s'articule autour de deux questions de recherche :

- Comment élaborer un processus qui serait moteur d'innovation s'appuyant sur une plateforme permettant la mise en réseau, le partage de compétences et de connaissances de l'ensemble des acteurs, à toutes les échelles de suivi de ces projets ?

- Comment fédérer l'ensemble des acteurs en matière d'aménagement urbain en vue d'une bonne gouvernance, par l'emploi d'outils numériques, libre et gratuits ?

Ainsi, l'hypothèse centrale de cette recherche est la suivante : l'approche Living lab permet la mise en place d'une gouvernance centrée sur l'usager, favorise l'innovation et s'avère particulièrement adaptée au développement local au travers de projets d'aménagements durables.

Pour ce faire, la démarche méthodologique proposée consiste à étudier les besoins des acteurs (usagers, publics, privés...) en vue de la réalisation de deux projets d'écoquartiers, en Lorraine, puis de proposer et mettre en place des outils numériques issus du Web 2.0, libre d'accès et d'utilisation, en open source ${ }^{19}$, mais aussi intuitifs et ergonomiques. Le premier projet s'intéresse aux processus d'aménagement urbain (objectifs, obstacles, enjeux économiques, sociaux, environnementaux...) auxquels sont confrontés les acteurs lorsque

\footnotetext{
${ }^{18}$ En distinguant l'écoquartier « perçu » (Gaudin, 2013), celui des grands projets urbains, sur le modèle des lois Grenelles, et l'écoquartier « idéal » (Renauld, 2012), afin de mettre en évidence les demandes et attentes des habitants.

${ }^{19}$ La désignation open source ou « code source ouvert » s'applique aux logiciels dont la licence respecte des critères précisément établis par l'Open Source Initiative, c'est-à-dire les possibilités de libre redistribution, d'accès au code source et de création de travaux dérivés. Mis à la disposition du grand public, ce code source est généralement le résultat d'une collaboration entre programmeurs.
} 
l'initiative provient de l'échelon le plus bas. Cette démarche dite ascendante (bottom-up) s'illustre par le projet d'écoquartier Ecolline, dans la commune de Saint-Dié-des-Vosges (département des Vosges), puisqu'il est à l'initiative d'une petite communauté de citoyens, organisée pour sa construction et réalisation. Le second projet permet d'étudier ces mêmes processus d'aménagement urbain, lorsqu'à l'inverse l'initiative vient d'un échelon supérieur. Cette démarche dite descendante (top-down) s'illustre par le projet d'écoquartier Hermitage, dans la commune de Joeuf (département de la Meurthe-et-Moselle), puisque l'initiative vient de la collectivité territoriale de Joeuf. Dans ces projets, des démarches participantes et d'observations ${ }^{20}$ sont réalisées et complétées par des entretiens semi-directifs ${ }^{21}$.

Ainsi, au travers de ces deux terrains d'études, l'approche Living Lab est proposée et adaptée en fonction des demandes et besoins des acteurs, en y intégrant des outils numériques, libres et gratuits, afin d'apporter un soutien technique pour une meilleure innovation, communication et gouvernance. Pour ce faire, il est indispensable d'identifier de manière exhaustive tous les acteurs pour leur mise en réseau, puis, de cerner les différents besoins et attentes des habitants. Des rencontres ont donc été organisées avec les représentants de quartiers et d'associations des deux terrains d'étude. Ensuite, des entretiens semi-directifs ont été menés auprès de l'ensemble des acteurs, et analysés à l'aide du logiciel Ethnos $^{22}$. Ces étapes permettent d'identifier précisément les besoins des acteurs en matière d'outils Web 2.0, pour la mise en place d'une bonne gouvernance. Enfin, d'un point de vue technique, une boîte à outils de logiciels libres est proposée pour la mise en place d'une plateforme communale SIG (Système d'Information Géographique) permettant de servir de support de communication entre tous les acteurs. D'une manière générale, cette plateforme permet aux habitants, par le biais de la géolocalisation, de transmettre directement leurs remarques vis-à-vis de la commune. Par exemple, ils peuvent positionner un problème technique avec un commentaire associé, localiser un événement associatif ou faire des propositions diverses. Cette plateforme constitue donc la moelle épinière de la gouvernance, car elle permet la rencontre de tous les acteurs et le partage d'information en temps réel. Toutefois, en se basant uniquement sur cette plateforme, cela entraine l'exclusion de la population ne maitrisant pas l'outil informatique ou n'étant pas équipée. Pour y pallier, la gouvernance prend également des formes moins virtuelles avec des journées de rencontre, des évènements culturels sur chacun des sites pour que les habitants puissent s'approprier et s'investir dans ces projets.

\footnotetext{
${ }^{20}$ Pour chacun des deux terrains d'étude, un stage de Master 2 Recherche en géographie, d'une période de 6 mois, a été réalisé en 2012, par un étudiant, Léo Casagrande, inscrit à l'Université de Lorraine, afin d'intégrer les comités de pilotage des deux projets d'écoquartiers.

${ }^{21}$ Des entretiens semi-directifs ont été conduits auprès d'acteurs publics et privés : Maires, Elus et Responsables des services urbanisme de Saint-Diè-des-Vosges et de Joeuf ; Elus territoriaux dans les départements des Vosges et de la Meurthe-et-Moselle ; Membres actifs et Responsables de l'association Ecolline ; Représentants des citoyens de l'Hermitage ; Autoconstructeurs de maisons écologique (label Bâtiment de Basse Consommation BBC) ; Autoconstructeurs de maisons en paille (BBC) ; Urbanistes ; Architectes ; Maîtres d'œuvre ; Paysagistes ; Ingénieurs ; Juristes ; Programmistes (la programmation architecturale et technique entre dans la catégorie des métiers d'assistance à la maîtrise d'ouvrage, puisque ce corps facilite le travail de l'architecte, avant la construction, il définit les espaces, les volumes, les circulations... pour que personnels et usagers apprécient les lieux de vie. D'après la fiche métier du site OuestFrance-emploi.com [URL : http://www.ouestfranceemploi.com/metiers/programmiste], consulté le 6 août 2014).

${ }^{22}$ Téléchargement gratuit du logiciel Ethnos, pour les étudiants et les enseignants, [URL : http://www.softconcept.com/blog/ethnos-gratuit/], consulté 17 juillet 2012.
} 
Le projet d'écoquartier Hermitage de la commune de Joeuf est intéressante tant il constitue un cas d'école en matière d'aménagement du territoire et de reconversion industrielle. En effet, le tissu urbain dense et typique de la ville enclavée, en fond de vallée, est essentiellement composé de cités ouvrières, vestiges du passé sidérurgique de la Lorraine. Le sous-sol communal, rendu instable par les effondrements d'anciennes galeries minières est soumis à un plan de prévention des risques qui contraint fortement la collectivité dans son expansion, pourtant nécessaire. Le seul terrain constructible est un ancien site industriel en friche, depuis la fermeture des dernières usines en 2004 (Europipe). Aujourd'hui, la municipalité souhaite reconvertir ce site en écoquartier pour y construire 490 logements ${ }^{23}$. Ce projet d'envergure s'inscrit dans la continuité d'une politique affirmée de développement durable de la part de la mairie. Les enjeux d'investir un tel projet sont d'observer comment une petite collectivité peut réagir et faire face à des problématiques telles que la dépollution d'un site industriel, l'instauration d'un dialogue et d'une concertation avec les usagers, la gestion des difficultés qui se pose à une équipe pluridisciplinaire autour d'un projet de développement durable. Ainsi, quelques pistes de réflexion seront apportées afin d'optimiser les résultats attendus par la collectivité. A l'inverse, puisque dans le cas de Joeuf, la décision de construire un écoquartier s'est faite en mairie, un organe décentralisé d'État, il est intéressant d'étudier la façon dont la décision du projet Ecolline s'est mise en place à l'initiative d'un groupe de citoyens. Dans ce cas, il s'agit de comprendre pourquoi les habitants-bâtisseurs ont décidés de se passer de l'impulsion politique inhérente à un projet de développement durable, pour devenir eux-mêmes les propres acteurs et aménageurs de leur territoire : quelles conditions, quels partenariats, quelles contraintes, quelles connaissances et quelle gouvernance ont-ils choisi ?

La première partie présente deux projets d'écoquartier permettant de comparer deux processus d'aménagement, où l'un est le choix de citoyens, et l'autre relève de la décision d'une collectivité territoriale. Suite à l'analyse des entretiens réalisés, la seconde partie permet de comprendre les attentes et besoins des acteurs, en matière d'innovation territoriale et de gouvernance, en vue de la réalisation des deux projets. La troisième partie s'attache à présenter les outils open source disponibles pour la mise en place d'une participation collective, notamment ceux accessible grâce au Web 2.0. Après une analyse critique de la démarche, la conclusion énonce les conditions en vue d'une bonne gouvernance.

\section{Ecoquartiers et constructions durables : autopromotion et gouvernance}

\subsection{L'écoquartier Ecolline : quand l'aménagement est le choix de citoyens}

Le projet Ecolline se situe dans la périphérie de la commune de Saint-Dié-des-Vosges, dans le département des Vosges, en région Lorraine. Ce projet a été initié sur un terrain constructible d'une superficie d'1,5 hectare, dans un cadre rural. Il s'agit d'un écolieu d'habitats groupés dont les membres fondateurs, constitué en autopromotion et autoconstruction, sont regroupés en association. Ce projet se compose de maisons bioclimatiques passives, pensées et élaborées par plusieurs familles lorraines et alsaciennes regroupées autour d'une charte de valeurs, dans un cadre naturel exceptionnel. Le

\footnotetext{
${ }^{23}$ Voir site de la mairie de Joeuf, Eco-quartier L'Hermitage, «Les données du diagnostic du SCOT» [URL : http://www.ville-joeuf.fr/fichiers/urbanisme/ecoquartier/hermitage.pdf], consulté le 18 juillet 2016.
} 
regroupement d'usagers en autoentrepreneurs ${ }^{24}$ permet de s'organiser et de se structurer afin de penser et donner forme à leur projet d'habitat. Ce projet s'inscrit en parallèle des circuits d'aménagement traditionnels, puisqu'elle permet d'être initiée : aux techniques d'écoconstruction, à la démarche d'autopromotion, d'observer le mode de gouvernance qui s'opère au sein d'un tel projet, tout en esquissant des pistes de réflexion quant aux attentes des citoyens en matière de développement durable (choix des matériaux de construction, performances énergétiques), enfin de cerner les obstacles face à un tel projet.

Le projet Ecolline illustre la nouvelle tendance, en France, selon laquelle les citoyens développent, en expérimentant une nouvelle façon d'habiter, leur territoire : il s'agit de repenser les pratiques, de partager de nouvelles expériences, de changer les comportements, d'inventer d'autres usages et de réapprendre à vivre ensemble. Ecolline n'est donc pas seulement un projet de construction durable, il s'agit aussi d'une expérience humaine. Issue d'une réflexion et d'une démarche collective, elle repose sur un " projet de vie en harmonie avec les idées de partage, de solidarité, d'échanges, de vie saine, de modifications des comportements de consommation et de respect de l'environnement » (extrait des statuts de l'association Ecolline). La dimension sociale transparait dans l'ensemble des entretiens réalisés : "Pour chacun, il a été question de faire un travail sur soi et vers les autres, trouver un équilibre et faire des compromis pour prendre des décisions à l'unanimité... $L$ 'écoute, la remise en question et la communication ouverte et non violente sont les garants de la réussite» (membre de l'association). La cohabitation est pensée et le projet articulé autour des espaces collectifs. Les habitants mutualisent certains espaces, tout comme les commandes de matériaux, les échanges de savoir-faire, et s'entraident dans la construction.

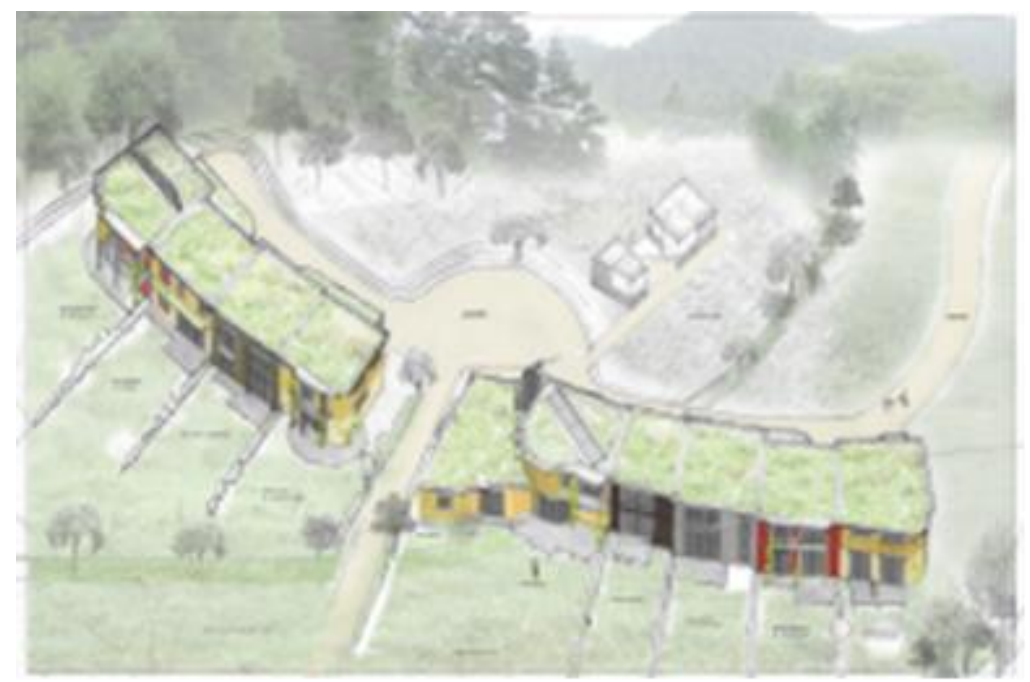

Figure 1: Le projet Écolline, à Saint-Diè-des-Vosges

Source : Ascendance Architecture, réalisé sous Sketchup)

L'enjeu du projet repose sur une mixité sociale en permettant de faire cohabiter une dizaine de familles dont les origines sont différentes. Le projet allie également une mixité

\footnotetext{
${ }^{24}$ Statut d'une personne qui crée une entreprise individuelle dépendant du régime fiscal et social de la microentreprise, afin d'exercer une activité commerciale, artisanale ou libérale.
} 
fonctionnelle, puisqu'outre les activités de l'association, un bureau d'études et un atelier de menuisier sont implantés, et certains habitants envisagent de travailler à domicile. Le projet Ecolline constitue un véritable modèle d'appropriation des quatre principes du développement durable (social, environnemental, économique et gouvernance) par des citoyens : « (...) des valeurs communes, mais c'est loin de suffire (...) Il y a vraiment une volonté, d'avancer ensemble, quel que soit le rythme, parce que tout le monde n'évolue pas à la même vitesse. (...) En prenant conscience de beaucoup de choses, que ce soit au niveau personnel et au niveau global, on a tous évolué d'une manière incroyable depuis le départ de ce projet » (membre de l'association Ecolline). Si la communication interne est cruciale au fonctionnement et au développement du projet, la communication et l'organisation du groupe vis-à-vis de l'extérieur le sont également. Il a donc fallu faire le choix d'une structure associative. Les partenaires institutionnels et les entreprises préfèrent travailler pour une structure, au statut clairement défini, plutôt qu'avec un petit groupe informel.

L'association 1901 Ecolline a vu le jour en septembre 2008 pour pouvoir représenter et structurer le groupe des futurs habitants de «l'Ecolieu ». A l'heure actuelle, sa forme est celle de toute association (président, trésorier, secrétaire), mais les membres souhaitent la faire évoluer vers une présidence collégiale, qui serait plus en phase avec leurs principes. La gouvernance est un des points forts du projet Ecolline. Toutes les décisions se prennent à l'unanimité, sauf en cas d'extrême urgence. L'unanimité dans un groupe d'une vingtaine de personnes est très difficile à obtenir, elle requiert une excellente concertation et une grande capacité d'écoute et de communication. L'association a, dès le début, engagé un dialogue avec les élus et partenaires institutionnels. De même, les relations avec les partenaires privés, comme les maîtres d'œuvre, sont des exemples de bonne communication. L'architecte s'est impliqué, a écouté, conseillé les habitants dans la conception du projet. «L'accompagnement de l'architecte, on peut dire qu'il a été au-delà de son rôle (...), parce que c'est vrai qu'il nous a souvent amenés à des pistes de réflexion » (membre de l'association Ecolline).

\subsection{L'écoquartier Hermitage : quand l'aménagement est le choix une collectivité territoriale}

Ce projet vise à créer un écoquartier sur une ancienne zone industrielle, constituant la dernière réserve foncière exploitable de la commune. Compte tenu des contraintes d'urbanisme qui pèsent sur le territoire communal (dont un aléa minier sur $95 \%$ du territoire, selon le Plan de Prévention des Risques Miniers ${ }^{25}$ ), et compte tenu du gel de la constructibilité qui a paralysé pendant dix ans le développement urbain. La création de ce projet constitue donc une expérience inédite pour les élus, comme pour les services municipaux.

L'écoquartier Hermitage est réalisé sur le site industriel Europipe, fermé en 2004. Ce site a une superficie de 18,5 hectares dont $35000 \mathrm{~m}^{2}$ de bâti. Il est situé à l'extrême est, du ban communal de Joeuf, dans le lieu-dit du Bas-de-Franchepré. La limite communale et départementale est matérialisée par la rivière l'Orne. Le site est donc, pour partie, en zone inondable. Il est classé en zone à urbaniser (1AU) dans le Plan Local d'Urbanisme ${ }^{26}$, prévoyant une occupation mixte du site (habitats et activités). Le site bénéficie d'une

\footnotetext{
${ }^{25}$ PPRM approuvé le 24 novembre 2009.

${ }^{26}$ PLU approuvé le 28 janvier 2008.
} 
situation privilégiée sur la commune, puisqu'il représente un attrait paysager indéniable, ouvert sur des éléments emblématiques (collines boisées, châteaux...). Les bords de l'Orne constituent des limites vertes, permettant un accès à un cadre naturel (ripisylves denses, espaces boisés). De plus, cet espace, accessible depuis toute la partie est de la Vallée de l'Orne, est connecté aux autres quartiers et au centre-ville. Le site, bien qu'historiquement industriel et privé, révèle des espaces naturels à préserver, dont l'existence est insoupçonnée des habitants du fait de l'interdiction d'accès au site. Le coût des travaux de viabilisation a été estimé, pour l'ensemble de la zone, à environ dix millions d'euros. Ces travaux comprennent les voiries, les cheminements, l'assainissement, les réseaux divers, l'éclairage public, le mobilier urbain, la signalisation, et l'aménagement d'espaces verts.

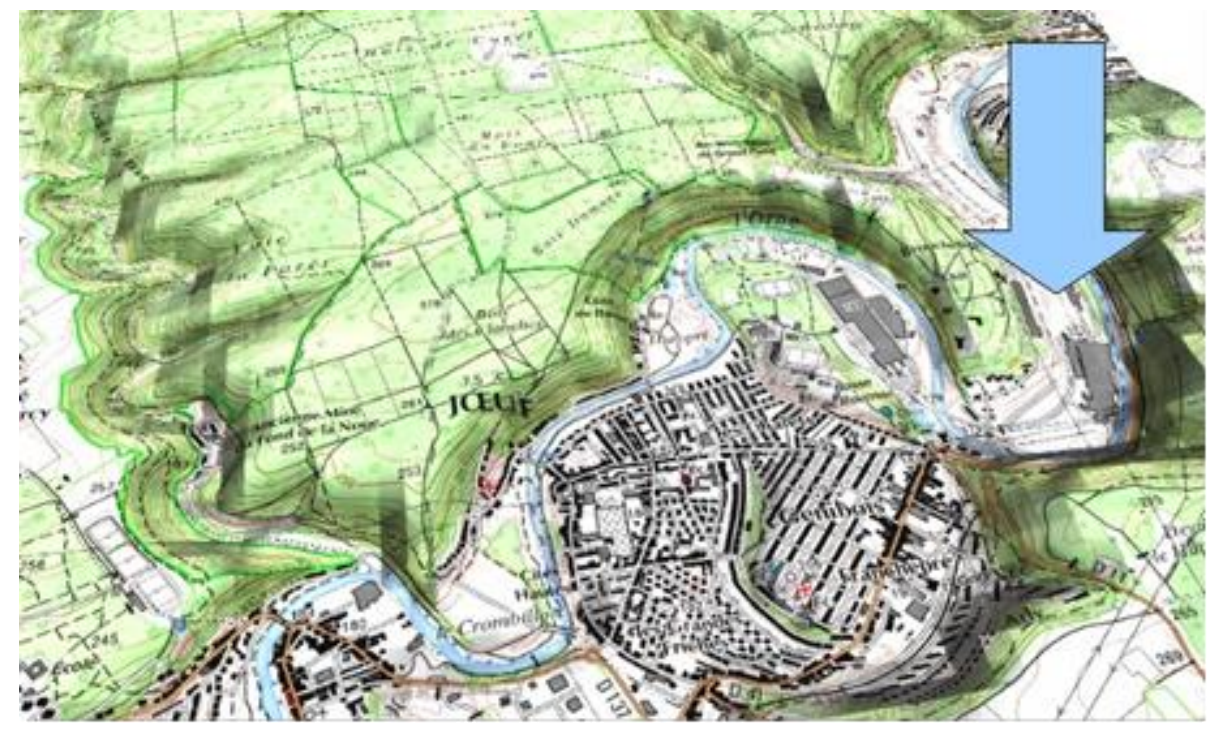

Figure 2 : L'implantation du projet Hermitage, à Joeuf

MNT réalisé avec Grass, à partir de données IGN, scan 25 et des données altitudinales du SRTM

La mairie de Joeuf souhaite faire de ce site « le lieu d'une démarche exemplaire et d'un projet d'aménagement urbain d'excellence, unique sur le territoire » (Maire de Joeuf). Ce projet-pilote doit en générer d'autres tout aussi innovants et permettre le développement d'emplois et de logements. En effet, le projet doit répondre quantitativement et qualitativement à une demande croissante de logements, en garantissant l'accès au logement familial des plus modestes et en privilégiant la mixité sociale, générationnelle, fonctionnelle, et des formes urbaines. L'enjeu de la gouvernance à Joeuf est de présenter le projet à la population, notamment les citoyens qui le souhaitent, en vue de leurs implications dans les différentes phases du projet, et les bailleurs sociaux qui se posent en partenaires clés du projet.

Néanmoins, la gouvernance se prépare largement en amont, bien avant de commencer la phase de mise en place. Le quartier de l'Hermitage est une ancienne zone industrielle, donc inhabitée et non utilisée par les habitants. En ce sens, celle-ci, bien que sur le territoire communal d'un point de vue administratif, ne fait « pas partie » de la ville dans les représentations mentales des habitants. De plus, lors de la description de la zone industrielle, 
des termes négatifs sont employés tels que : « néfaste », « sale », « polluée », etc. L’enjeu pour la commune est avant tout que les joviciens s'approprient le site.

\section{Les attentes et besoins des acteurs : innovation territoriale, modélisation et gouvernance}

L'objectif de cette partie est de cerner les attentes et besoins des acteurs, en matière d'innovation territoriale, de gouvernance, afin de leur proposer les outils du Web 2.0, les plus pertinents, pour la réalisation des deux projets d'écoquartier. Deux axes de recherche structurent l'analyse des entretiens : le premier, s'attache à diminuer les oppositions entre les réseaux linéaires et les territoires surfaciques afin que les acteurs puissent communiquer, échanger, car « réseaux et territoires sont dans le même rapport : l'espace géographique, qui les inclut tous les deux »(Brunet, 1993 : 480-481) ; le second concerne la mise en place d'outils de partage de l'information en temps réel qui permet les échanges entre les acteurs au sein d'un projet de territoire, en facilite la gestion, la conception, ainsi que l'aide à la décision. A l'issue de l'analyse des entretiens, huit éléments dominants ont été mis en évidence lors de leur dépouillement à l'aide du logiciel de traitement d'enquêtes Ethnos ${ }^{27}$ :

1. l'échelon local apparait comme le plus pertinent dans la mise en place un aménagement du territoire adapté, notamment par l'intermédiaire du Schéma de Cohérence Territoriale (SCOT) : outil de conception et de mise en œuvre d'une planification intercommunale, dont les avantages ont été évoqués par l'ensemble des acteurs institutionnels : « à une échelle trop grande, nous n'arrivons plus à impliquer tout le monde, et les gens ne se sentent plus concernés. Et à une échelle trop petite ça devient du nombrilisme » (Président de l'association Ecolline).

2. dans les projets d'aménagement, la participation collective est encouragée autant par les citoyens que par les collectivités. Le rôle des bailleurs sociaux est également mis en avant afin de favoriser l'autopromotion : « il y a surtout un boulot énorme avec les bailleurs sociaux pour, même au niveau de la location, trouver les formules où même si ce n'est pas complètement de l'autopromotion, les gens pourraient quand même participer à la construction et donc la respecter plus après. Les gens qui le font en général ont des bons résultats. " (Responsable de l'association Ecolline). De plus, l'absence de participation est responsable de l'échec de certains projets : la favoriser permet d'éviter de nombreux conflits : « le fait de dire, on a monté ce projet, il va y avoir ça à côté de chez vous, c'est ferme et définitif, vous n'avez rien à dire. Là c'est clair qu'il y aurait des conflits » (Chargé de l'urbanisme à Joeuf) ;

3. le Web 2.0 et les nouvelles technologies représentent, à l'unanimité des acteurs rencontrés, les outils de communication et de travail collaboratif indispensables et incontournables à tous projets d'aménagement, car ils facilitent les échanges, le partage de l'information et la collaboration à distance : « on n'aurait jamais pu autant avancer sans ça : puisqu'on communique avec notre architecte, notre thermicien et entre nous. Et on communique beaucoup avec les réseaux extérieurs, des gens sensibles aux mêmes choses

\footnotetext{
${ }^{27}$ Ethnos est un logiciel de conception et de traitement d'enquêtes. Il se distingue par son ergonomie et par une couverture fonctionnelle : il peut gérer de manière intégrée toutes les phases de l'enquête, de la rédaction du questionnaire et sa mise en page jusqu'à la mise en forme du rapport final. Voir site Softconcept [URL : http://www.soft-concept.com/logiciel-ethnos/], consulté le 23 juillet 2016.
} 
que nous, pour recruter des bénévoles, pour échanger des informations » (Responsable de l'association Ecolline) ; « dans notre travail, évidemment c'est indispensable » (Responsable du service urbanisme à Joeuf) ;

4. la question de l'innovation territoriale fait elle aussi l'unanimité : à l'inverse de la DATAR qui considère l'innovation territoriale sous un prisme économique (Lacour, 2010), les personnes rencontrées l'envisagent, avant tout, dans sa dimension sociale : " c'est effectivement l'aspect collectif et autopromotion, autoconstruction. Les gens qui sont là ont choisi la façon dont ils vont vivre, ensemble... » (Membre de l'association Ecolline) ; « Ce n'est pas forcément juste l'innovation dans les énergies renouvelables, même si c'est important aussi parce qu'il faudra au niveau des communes y réfléchir. L'aspect social c'est vraiment important » (Responsable urbanisme Joeuf) ; «L'innovation territoriale à mon sens, c'est l'innovation sociale, d'abord. » (Elu communal à Saint-Diè-des-Vosges) ;

5. si la grande majorité des personnes interrogées demeurent sceptiques face au concept du développement durable, qualifié de « galvaudé », « récupéré » politiquement, une minorité d'acteurs pense, qu'à condition de changer les modes de pensées, il constitue une bonne alternative aux crises de la société : «c'est un idéal de société, mais il faut vraiment changer la donne et l'esprit des gens » (Membres de l'association Ecolline). Le volet humain se démarque en premier. Quelle que soit la dimension perçue du développement durable, la tendance observée est celle d'un retour au local : « Il faut tout miser sur le local, toutes les ressources locales » (Chargée de communication à Ecolline);

6. concernant les concepts « ville » et « écoquartier », la dimension du lien social est la plus citée : la création du lien social est l'un des enjeux majeurs pour tendre vers une ville idéale. Ainsi, la création du lien social est une fonction essentielle pour une ville, plus encore que la mobilité et la proximité. Deux types de réponses majoritaires définissent idéalement un écoquartier : le «bien-être » et le « lien social », avant la «dimension environnementale » : «Un quartier qui fait du bien à la nature, qui n'est pas simplement dans le respect de l'environnement, mais qui produit de l'effet positif. Et pas simplement au plan énergétique » (Elu territorial à Joeuf). Si les réponses à la notion d'écoquartier sont plutôt positives, celles sur les projets mis en place par des grandes villes sont beaucoup plus critiqués : «c'est du greenwashing $^{28}$ (...) Ça parlait beaucoup de l'aspect des bâtiments et puis éventuellement des activités qu'il y aurait sur place, mais ça ne parlait pas du tout de la manière dont les gens pouvaient se rencontrer, discuter...» (Maire de Joeuf). De plus, dans l'opinion, ces réalisations, encouragées par les lois Grenelles, restent perçues comme étant peu accessibles à la majorité de la population et réservées aux catégories sociales plus élevées.

7. les politiques d'aménagement du territoire sont « mal perçues », « mal pensées », « semblent obsolètes » pour $40 \%$ des acteurs rencontrés, notamment, en raison de la place prépondérante laissée à l'automobile et des répercutions sur l'aménagement urbain. En revanche, l'importance du « lien social » apparait comme un élément important : « l'aménagement du territoire ne doit plus seulement être orienté selon une logique de

\footnotetext{
${ }^{28}$ Le terme anglais qui se traduit par «blanchiment vert ». D'après Le grand dictionnaire terminologique, le terme français vient de la contraction des mots green, vert et whitewash, littéralement blanchir à la chaux. Terme parfois utilisé pour désigner le blanchiment d'argent. Il est employé à la fin des années 1980. L'écoblanchiment (ou blanchiment écologique) est un procédé de marketing employé par une organisation (entreprise, gouvernement...) dans le but de donner à l'opinion publique une image écologique responsable : dans bien des cas, l'argent est davantage investi en publicité « verte » (la couleur symbolisant l'écologie), plus que pour de réelles actions en faveur de l'environnement.
} 
communication ou de productivité, mais en véritable vecteur de lien social » (Maire de SaintDiè-des-Vosges). De plus, il apparait que « la lutte contre l'étalement urbain » préoccupe l'ensemble des acteurs : " on a assisté à un aménagement du territoire qui est lui-même marqué par ce productivisme, que ce soit les grandes artères et les structures de communication, par voie fluviale, ferrée, terrestre, aérienne... On a d'abord réfléchi à être le plus utile pour l'industrie et pour notre économie » (Elu territorial à Joeuf).

8. la « gouvernance territoriale » est majoritairement définie comme le fait d'inclure une participation citoyenne dans les processus d'aménagement : " c'est vraiment la démocratie au sens propre. On consulte les gens sur leurs besoins, leurs envies, et puis on compte aussi sur eux pour la mise en œuvre... Enfin, c'est quelque chose de très participatif » (Président de l'association Ecolline) ; «Le principe fondateur, ça doit être justement la conviction que l'on doit faire avec ceux qui sont concernés et ceux qui sont intéressés » (Elu territorial à Joeuf).

Suite à l'analyse de l'ensemble des entretiens réalisés, il semble opportun, dans le but d'une bonne gouvernance, de mettre à disposition des acteurs des outils open source, leur permettant d'être connectés entre eux et avec leur territoire. L'innovation territoriale, n'est possible que dans la mesure où tous les acteurs, organisés en réseau, communiquent efficacement et disposent d'outils adaptés. Les champs d'application sont multiples : internet, réseaux communautaires, réalité augmentée... Tous ces outils enrichissent les territoires d'informations et permettent une plus grande mobilité des données et des acteurs. Ainsi, la ville 2.0 offre une plateforme d'innovation ouverte, afin de garantir son développement économique, social, culturel et de tendre vers une véritable cohésion territoriale dans un système où pour se développer chaque territoire doit rester compétitif (Kaplan, Marcou, 2009).

\section{L'apport du SIG et de la 3D dans la conception des projets d'aménagement}

Les outils présentés ci-après permettent de répondre aux attentes et besoins des acteurs pour la mise en place d'une bonne gouvernance. Cette partie n'a pas la prétention d'être exhaustive $^{29}$, mais elle constitue une boite à outils open source, en vue de la planification, de la mobilisation et de l'apport des logiciels en libre-service du Web 2.0.

\subsection{Web 2.0, un modèle de gouvernance}

Le Web 2.0 est à la fois une innovation technologique et une nouvelle forme de gouvernance (Fayon, 2010), comme en atteste l'avènement de nouveaux usages, plateformes et outils (réseaux sociaux privés et professionnels, sites d'échanges, de partage d'informations, de données...). Ces applications ont eu un tel impact sur les pratiques, les usages, l'accessibilité et le partage des données en temps réel, que l'on peut en ressentir les effets, directs ou indirects, sur les territoires (Musso et al., 2002). Si ces effets sont qualifiés de palimpsestes (Eychenne, 2009), tant ils portent en eux les traces des pratiques anciennes et contemporaines, il en est de même pour le Web, constitué d'informations superposées les unes sur les autres à l'infini.

\footnotetext{
${ }^{29}$ Voir l'ouvrage de Vergnet-Covo (2009).
} 
Les réseaux sociaux et de partage (Facebook, Twitter, Instagram, Linkedin, Viadeo, Dailymotion, Wikipédia...) ont permis à leurs utilisateurs d'échanger des informations, des données, des avis, des conseils, d'entretenir un réseau privé et professionnel avec d'autres utilisateurs et acteurs. En effet, ces utilisateurs ne se contentent plus d'utiliser les ressources d'Internet, mais ils contribuent à alimenter la toile en diverses informations qui ont un impact direct dans la vie quotidienne. Internet permet donc aux secteurs public et privé de communiquer et de recueillir les avis des utilisateurs et de les informer sur les services proposés (la SNCF «twitte»en temps réel pour informer les passagers d'éventuelles perturbations sur le réseau ferroviaire). Internet permet aussi, au tiers secteur, de partager et de rechercher toute l'information dont il a besoin et de collaborer dans l'élaboration de nouvelles plateformes et services (Dismoioù.com, Justeacote.com, Qype.fr) qui informent sur les horaires des transports en commun, permettent de donner un avis sur un produit, un lieu, un professionnel, et des conseils en tout genre.

Le Web 2.0 est un modèle de participation collective qui place l'usager au centre de ses processus. A l'origine de la transformation du Web en Web 2.0, on retrouve bon nombre de plateformes collaboratives où l'utilisateur agrémente lui-même la base de données. L'avènement de l'Internet mobile, des systèmes GPS embarqués (Global Positionning System) et de la géolocalisation des smartphones ouvre une nouvelle porte vers un monde où l'information géographique se partage en temps réel, mais surtout, se spatialise d'elle-même. Plus besoin d'être spécialiste ou expert en géomatique pour alimenter une base de données cartographique. D'ailleurs, de plus en plus d'applications, comme Facebook, utilisent la géolocalisation et la cartographie pour représenter leurs informations. L'information géographique tend donc à se démocratiser en devenant accessible au plus grand nombre. La carte, formidable outil géographique, est-elle en train de devenir le support incontournable de l'information de demain? Pour Lévy (2011) "le numérique permet de faire des cartes d'un nouveau genre, de type séquentiel : on ne représente pas une situation à l'instant " $t$ ", mais les dynamiques d'évolution d'un territoire ou d'un tout autre phénomène à composante géographique, comme l'espace d'une ville. Le paradoxe est que ce renouvellement de la cartographie s'inscrit dans un contexte où l'éducation à la carte est très faible. (...) Or, dans la perspective d'une démocratie participative autour des projets d'aménagement, il importe que les participants sachent décrypter les cartes, à commencer par celles produites par les urbanistes ».

\subsection{La carte, une interface et une infrastructure}

La carte est devenue un support quasi incontournable de l'information sur le Web 2.0. Par exemple, la plateforme Google Map offre une représentation précise du territoire, et l'option « street view » illustre les rues à l'aide de photos. Les fonds de cartes Google Map sont repris et utilisés par des institutions, des entreprises et des usagers pour y ajouter des informations pratiques. Suite à cette démocratisation de la carte et du plan, il est concevable que le géographe soit perplexe face à ces changements de pratiques et aux risques de voir son expertise délaissée. Pourtant, le travail de géomatique n'est pas destiné à disparaître, au contraire, il est très intéressant que l'usager lambda puisse apprendre à lire et à écrire sur les cartes. Cela ne remplace pas le travail du géographe, mais contribue seulement à créer un nouveau type de carte, qui « ouvre la voie à l'émergence de nouveaux services urbains... et modifie, dans des proportions encore mal connues, la manière dont les citadins interagissent 
avec leur ville » (Eychenne, 2009). Une fois de plus, la carte, envisagée comme un outil de concertation, d'interaction et de participation citoyenne, ouvre quelques pistes vers une nouvelle forme de gouvernance appliquée aux territoires.

Pour Eychenne (2009) « là où la géomatique traditionnelle recherche la précision, la rigueur et la lisibilité au travers d'une approche très formalisée portée par des professionnels, la néo-géographie reposerait presque sur le contraire. L'abondance de données en compenserait l'imprécision ou la disparité. La simplicité et l'ouverture des outils, qui permet de multiplier les représentations et les applications, feraient émerger a posteriori des connaissances et des pistes d'action qu'une approche analytique ne saurait pas voir ». Toutefois, les objectifs ne sont pas les mêmes : la géomatique et les SIG analysent le territoire et fournissent des supports précis de l'information pour la décision, la gestion ou la conception de projets, la cartographie 2.0, quant à elle, se contente simplement d'afficher et d'organiser les informations avec une approche spatiale. Il est donc nécessaire d'accompagner cette démarche, de partager notre savoir et de faire évoluer ces pratiques pour ne pas rester en marge d'un mouvement étonnant porté par les usagers. De plus, cette démarche permet de recueillir nombre d'informations utiles afin d'observer et d'analyser les liens directs, méconnus, entre les citoyens, leurs pratiques et leurs territoires. De ce fait, les SIG s'avèrent utile et efficace dans le traitement des informations.

\subsection{Les SIG au centre du traitement de l'information de demain}

En plus des données cartographiques numériques que constituent le Web 2.0, la ville 2.0 regorgent de capteurs qui collectent une masse d'informations : météo, qualité de l'air, trafic autoroutier, vidéosurveillance... Ces informations, massives et brutes, sont autant de données à traiter qui n'apparaissent cohérentes qu'une fois analysées et spatialisées. Ces données brutes manquent souvent de structure et de lisibilité, c'est donc en s'attachant à développer des outils qui permettent de les mettre en relation et d'effectuer les traitements spatiaux et analytiques adéquats, qu'il est possible de les rendre intelligibles. Ainsi, l'utilisation du SIG prend tout son sens.

Les SIG offrent toutes les possibilités de traitements relatifs aux systèmes de bases de données et permettent une visualisation cartographique. Parce qu'ils permettent l'analyse d'informations relatives aux enjeux majeurs de notre société (environnement, démographie, santé, aménagement du territoire), les SIG sont un outil incontournable dans la planification et la gestion des territoires.

\subsection{La 3D, un outil de conception, d'aide à la décision, de communication et de gouvernance}

L'outil de conception tridimensionnelle ou 3D, se démocratise à la fin des années quatre-vingt-dix, et équipent les ordinateurs domestiques. La modélisation 3D, qui permet la modélisation et la simulation en réalité virtuelle, est devenue un outil d'aide à la communication et à la décision. Par exemple, la simulation d'une crue permet aux élus de décider des zones à classer urbanisables ou non. La vision d'un bâtiment dans le paysage aide dans le processus de décisions. La 3D permet également une interactivité entre décideurs et usagers. Par exemple, les habitants d'une commune peuvent effectuer une visite virtuelle d'un projet et ainsi donner leur avis en vue de l'améliorer. La 3D, par l'image 
complète et quasi réelle qu'elle renvoie, permet une meilleure compréhension et lisibilité du projet, puis d'en modifier les contours à l'infini en temps réel. Elle est donc un outil de gouvernance indissociable des projets d'aménagement du territoire.

\subsection{CAO, SIG et Webmapping, la boite à outils open source}

L'alternative open source permet, à moindre coût, d'appréhender différemment le traitement de l'information géographique, dont nombre de services et d'entreprises peuvent tirer profit. L'utilisation de l'open source ne génère que les frais liés à la formation. De plus, les suites SIG open source, sont tout aussi efficaces que les suites propriétaires (MapInfo, Arcgis, Geoconcept), tout en offrant un format de données compatible avec les suites propriétaires, grâce notamment à l'uniformisation des formats de données géographiques liées à l'Open Geospatial Consortium (OGC).

Les objectifs affichés sur le site de l'OGC $^{30}$ sont de regrouper l'ensemble des acteurs concernés par la manipulation de données géographiques afin d'élaborer des standards ouverts garantissant l'interopérabilité des données, puis de favoriser la coopération entre les développeurs, fournisseurs et utilisateurs dans les domaines des SIG et de la géomatique. Parmi les formats recommandés par l'OGC, le langage GML (Geography Markup Language), dérivé du langage internet $\mathrm{XML}^{31}$ (Extensible Markup Language) est spécialement adapté pour les données géographiques. Ce format ouvert permet de construire des modèles de données spécifiques dans les domaines comme l'urbanisme, l'hydrologie, la géologie... Ce langage, très utile en SIG, permet de décrire les formes d'objet, les projections, la topologie, le temps, les unités de mesure et les attributs de chaque objet géographique. Le GML, avec le format KML (Keyhole Markup Language), est utilisé pour la gestion de l'affichage de données géospatiales dans les logiciels de SIG, tel que Google Earth. L'utiliser permet une interopérabilité entre tout SIG et Google Earth, autant pour y exporter et publier des données sur le web que pour importer des informations à partir de cette plateforme.

Un autre format remarquable issu de l'OGC est le langage WMS (Web Map Service) qui permet de produire des cartes à partir de différents serveurs de données. Ce format est très pratique, car en plus d'une excellente intégration avec les suites SIG, il permet également d'éditer et de consulter des cartes sur le web, directement à partir d'un navigateur via la barre d'adresse URL. En pratique, une requête WMS permet de renvoyer à l'utilisateur une image visualisable sur un écran d'ordinateur après connexion à un serveur qui produit une image aux formats JPEG (Joint Photographic Experts Group) ou SVG (Scalable Vector Graphics).

Pour illustrer les possibilités de l'open source en vue d'un projet d'aménagement, une boite à outils peut-être utile à tous. Les exemples suivants donne un aperçu des possibilités de l'open source : de la constitution, gestion de la base de données, des SIG à la modélisation $3 \mathrm{D}$, jusqu'à la publication de cartes sur des plateformes en ligne.

\footnotetext{
${ }^{30}$ Voir site de l'OGC [URL : http://www.opengeospatial.org/], consulté le 26 juillet 2016.

${ }^{31}$ Extensible Markup Language qui se traduit par «langage de balisage extensible ».
} 


\subsubsection{Base de données : PostgreSQL et son extension géographique PostGIS}

PostgreSQL ${ }^{32}$ est un Système de Gestion de Base de Données relationnelles (SGBD) open source fonctionnant avec un langage SQL (Structured Query Language, soit Langage de requêtes structurée). Les avantages sont nombreux quant à l'utilisation de PostgreSQL dans la constitution d'une base de données géomatiques. Il agit comme un serveur pouvant avoir des relations avec des usagers informatiques, les clients. Cela s'avère pratique lors d'une utilisation entre plusieurs partenaires (en interne ou en externe) souhaitant collaborer et travailler sur une même base de données. PostgreSQL fonctionne sur les principaux systèmes d'exploitation et traite tous types de données (entiers, caractères, fonctions...). L'extension PostGIS ${ }^{33}$ permet de joindre des informations géographiques et géométriques aux données contenues dans la base (forme, projection, emprise, couleur, taille...).

En effet, la première étape du géomaticien dans la mise en place du SIG est la constitution de la base de données. Certaines suites propriétaires de SIG comme « ArcGis » possèdent leur propre système de gestion de base de données (comme ArcCatalog). A première vue cela peut sembler plus simple et plus efficace de gérer et de traiter les données avec le même logiciel. Cependant, il existe de nombreux avantages à utiliser PostgreSQL et ce en toute intégration avec les outils de traitement SIG, ce qui constitue tout l'intérêt de l'open source. L'intégration de PostgreSQL aux SIG libres permet de consulter la base directement sous l'interface SIG et d'exporter les données issues de ce SIG directement dans PostgreSQL : les informations utiles sont donc adressées à PostGIS. Cela permet également à chaque partenaire de travailler sur des données mises à jour en temps réel. Par exemple, si le fichier client d'une entreprise est réalisé sous PostgreSQL, le géomaticien transformera la base de données en SIG et ainsi spatialisera toutes les informations qu'elle contient. On peut alors repérer sur une carte tous les acteurs, sélectionnés via une requête SQL, et prévoir le meilleur itinéraire pour les rencontrer. Cela permet également à chaque partenaire de travailler sur des données mises à jour en temps réel, même si ceux-ci sont à des centaines de kilomètres. Cette manipulation peut sembler à première vue complexe, mais elle s'avère très utile, par exemple lorsque le fichier client d'une entreprise est fait avec PostgreSQL : il devient alors plus simple de transformer la base de données en SIG, ainsi de spatialiser toutes les informations qu'elle contient, ce qui permet de repérer sur une carte tous les clients, de sélectionner via une requête SQL ceux qu'il faut rencontrer dans la journée et ainsi prévoir le meilleur itinéraire pour relier tous les clients.

\subsubsection{Cartographie et SIG : le binôme Grass GIS et QuantumGIS}

Grass GIS ${ }^{34}$, Geographic Ressources Analysis Support System, est un outil SIG open source, complet et puissant ${ }^{35}$. Il permet la digitalisation de données et le traitement d'images

\footnotetext{
${ }^{32}$ Lien de téléchargement de PostgreSQL, [URL : http://www.Postgresql.org/download], consulté le 26 juin 2013. Des tutoriels aident à utiliser PostgreSQL, [URL : http://www.davidgis.fr/documentation/win32/html/index.html], consulté le 26 juin 2013.

${ }_{33}^{33}$ PostGIS est la contraction de Postgre et de Geographic Information System (SIG en français).

${ }^{34}$ Grass GIS signifie Geographic Ressources Analysis Support System Geographic Information System. Il s'agit actuellement de l'outil SIG le plus complet et le plus puissant accessible gratuitement et en open source. Lien de téléchargement, [URL : http://grass.fbk.eu/download/index.php], consulté le 26 juin 2013.
} 
satellites, de données «raster» (tous les formats d'images existants), de fichiers vectoriels (tous types de formats à l'import et à l'export: «.tab», «.shp»), toutes les analyses statistiques. De plus, il possède un module de visualisation des surfaces en trois dimensions (nommé NVIZ Visionneuse 3D) pouvant générer des MNT (Modèles Numériques de Terrain) en intégrant même le bâti (import des fichiers Autocad «.dxf »...) et d'en exporter une séquence animée.

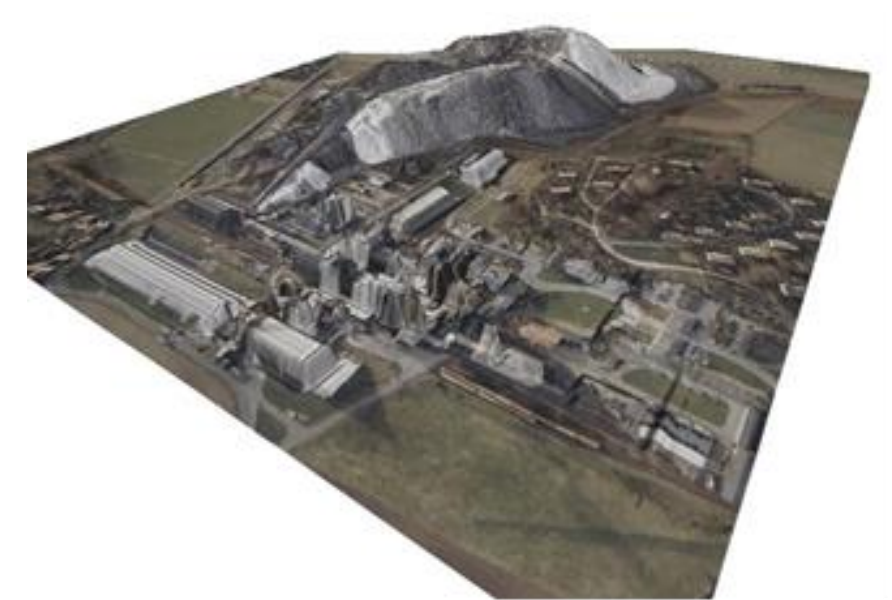

Figure 3: Extrusion des bâtiments à partir d'une orthophotographie, réalisée sous Grass GIS Source : Grass

A partir d'un MNT et des paramètres hydrologiques de débits, Grass est capable de modéliser un écoulement et d'en prévoir la crue en cas de précipitations importantes. Le logiciel reconnaît lui-même, d'après la géologie et l'altitude, le tracé du cours d'eau et intègre parfaitement les bâtiments 3D de la ville soumise à l'aléa.

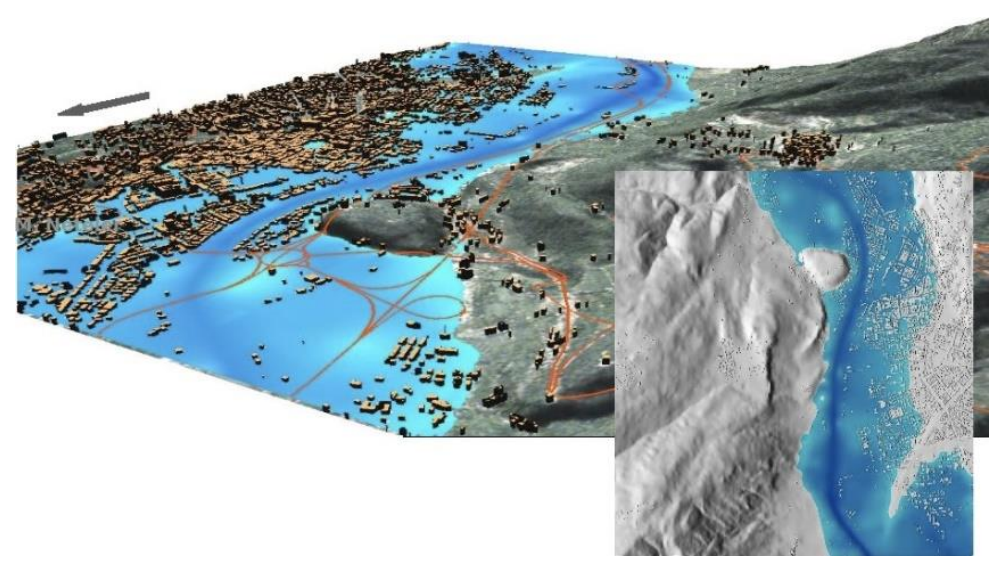

Figure 4 : Modélisation 3D d'une ville et simulation d'un épisode de crue, réalisée sous Grass GIS Source : Grass

\footnotetext{
${ }^{35}$ GrassGIS est aujourd'hui utilisé par la NASA, la NOAA, le USDA, l'US National Park Service, ainsi que plusieurs universités et collectivités européennes... [URL : http://grass.fbk.eu/download/index.php], consulté le 26 juin 2013.
} 
La prise en main de Grass repose en partie sur la capacité de l'utilisateur à maitriser les lignes de commandes. Pour pallier cette difficulté, il est possible de l'intégrer à un autre logiciel libre : QuantumGIS, dont l'interface est plus ergonomique.

Le logiciel de SIG, libre, QuantumGIS (QGis) est moins performant que Grass, mais présente une interface graphique, sans lignes de commandes, pour l'utilisateur. Il permet de gérer l'extension spatiale de Postgre, PostGIS, d'intégrer la lecture et l'écriture de données GPS. Il possède également un module de géoréférencement et intègre les fichiers Shapes $(. s h p)^{36}$. Qgis fournit une interface graphique conviviale à l'utilisateur en lui permettant d'avoir accès à toutes les puissantes fonctionnalités de Grass.

L'utilisation de ces deux outils permet de disposer d'une suite de logiciels libres pour la mise en place, gratuitement, de tout SIG. De plus, avec Qgis l'intégration des données traitées dans PostgreSQL, via PostGIS, permet de relier la base de données principale au SIG et de la mettre à jour à chaque fin de session de travail. Ainsi, après avoir présenté l'utilisation de logiciels open source et leur contribution au traitement et au partage de données, la partie diffusion et mise en réseau de ces données est abordée.

\section{Serveurs cartographiques et application cartographique : Map Guide}

Le Webmapping, ou cartographie sur le net, est une des innovations du Web 2.0. Le logiciel Map Guide ${ }^{37}$ est une solution de Webmapping en open source, où l'utilisateur peut mettre en place un serveur cartographique consultable et éditable en ligne, sans connaissance particulière en langage de programmation. Map Guide permet d'intégrer les données issues du traitement SIG de QGis-Grass et de les publier de manière interactive sur le web. Il est donc intéressant de savoir manier cet outil afin de pouvoir publier de véritables portails cartographiques en ligne.

Dans le cas d'un projet d'aménagement, il peut s'avérer utile, pour les différents acteurs, de pouvoir accéder à des documents numérisés (cadastres, scan $25^{38}$, photos aériennes, PLU, projets...). Il en sera de même dans la communication avec les citoyens qui pourront suivre l'évolution du projet et émettre des suggestions et propositions. Aussi, les avantages à utiliser Map Guide open source sont :

- l'accès aux données spatiales d'aide à la décision, de conception, que l'on soit à l'intérieur ou à l'extérieur de la structure, grâce au web (collectivité, entreprise) ;

- les manipulations de cartes : faciles et ergonomiques ;

- l'installation de la solution et de déploiement ;

- les outils de gestion et de publication de cartes (sans lignes de commandes);

- l'interopérabilité avec tous les formats standards de cartographie, conformes aux normes de l'OGC et l'intégration des données des utilisateurs ;

- les options de développement grâce au libre accès aux codes sources ;

\footnotetext{
${ }^{36}$ Les fichiers Shapes, ou fichier de formes, est un format de fichier issu du monde des SIG. Initialement développé par ESRI (Environmental Systems Research Institute). Ce format est largement utilisé par un grand nombre de logiciels libres (MapServer, Grass, QGIS...) et propriétaires (Autodesk, Map 3D...).

${ }^{37}$ Lien de téléchargement du logiciel libre «Map Guide », [URL : http://mapguide.osgeo.org/download], consulté le 26 juin 2013.

${ }^{38}$ Le scan 25 est une image numérique continue sur tout le territoire français des cartes de l'Institut national de l'information géographique et forestière (IGN) au 1/25 000 .
} 
- la gratuité d'utilisation.

Après avoir présenté la mise en place d'une base de données orientée SIG (Postgre, PostGIS), puis les outils pour une représentation en 2D et 3D (Grass GIS, QGis), puis la publication de ces données sur une plateforme cartographique (Map Guide), la dernière étape consiste à pouvoir générer des modèles en 3 dimensions des bâtiments.

\section{Modélisation 3D : Blender et Google Sketchup}

Blender et Google Sketchup constituent deux logiciels utiles à la modélisation en trois dimensions. En effet, la modélisation 3D détaillée est un formidable outil d'aide à la décision et de communication en matière d'aménagement du territoire. Si Grass permet de générer les MNT qui servent de supports à la modélisation, les deux logiciels suivants permettent de créer des formes et des objets qui viennent s'implanter sur le MNT.

La stabilité et les performances du logiciel Blender ${ }^{39}$ sont équivalentes à celles de logiciels propriétaires de Conception Assistée par Ordinateur $(\mathrm{CAO})^{40}$ et de Dessin Assisté par Ordinateur $(\mathrm{DAO})^{41}$. Ses applications vont de la conception d'objets 3D, de films d'animation, et de jeux vidéo (modélisation, rendu en $3 \mathrm{D}$, animation). Blender permet, à partir d'un environnement virtuel, de générer un programme exécutable (format « .exe ») où l'utilisateur évolue à l'aide des touches directionnelles du clavier, et interagir avec les éléments du projet d'aménagement.

\footnotetext{
${ }^{39}$ Lien de téléchargement du logiciel libre «Blender », [URL : http://www.blender.org/download/getblender], consulté le 26 juin 2013.

${ }^{40} \mathrm{La}$ CAO comprend l'ensemble des logiciels et des techniques de modélisation géométrique permettant de concevoir, de tester virtuellement - à l'aide d'un ordinateur et des techniques de simulation numérique - et de réaliser des produits manufacturés et les outils pour les fabriquer. Elle n'a pas pour fonction première l'édition du dessin. Elle désigne principalement du dessin en $2 \mathrm{D}$ et 3D : calcul de résistance, optimisation de l'utilisation des matériaux, pilotage de machines-outils et robots, calcul de coûts de fabrication, etc. La CAO permet ensuite la simulation de comportement de l'objet conçu.

${ }^{41}$ Le DAO permet de produire des dessins techniques avec un logiciel informatique. Il ne permet pas l'interprétation technique de l'ensemble et n'est pas exclusivement orienté vers l'industrie, mais aussi vers l'art. On le distingue de la synthèse d'image dans la mesure où il ne s'agit pas du calcul de rendu d'un modèle numérique, mais de l'exécution de commandes graphiques (traits, formes diverses...). En $\mathrm{DAO}$, la souris et le clavier remplacent le crayon et les autres instruments du dessinateur.
} 


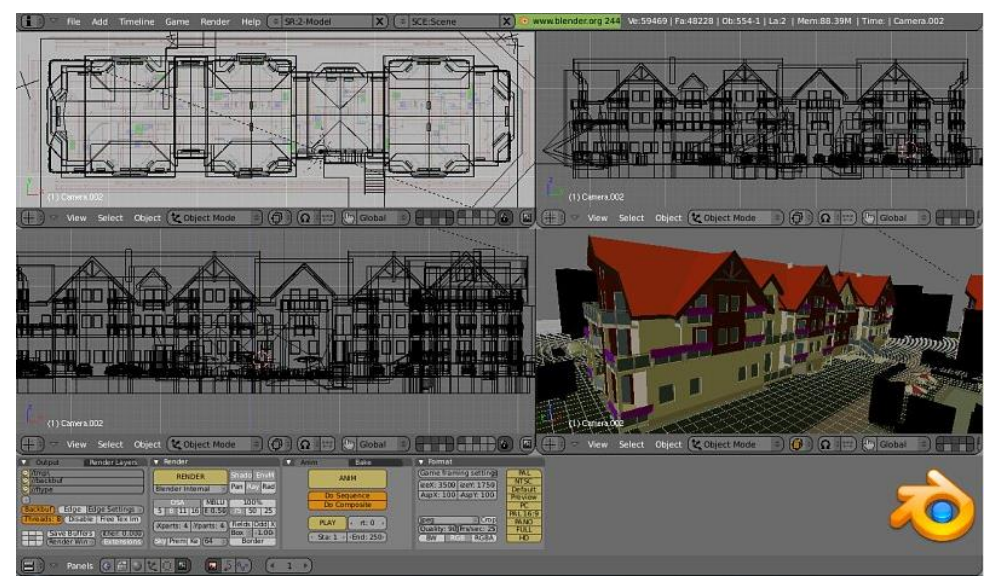

Figure 5 : Exemple d'architecture modélisée à partir du logiciel Blender

Source : K. Nowacki

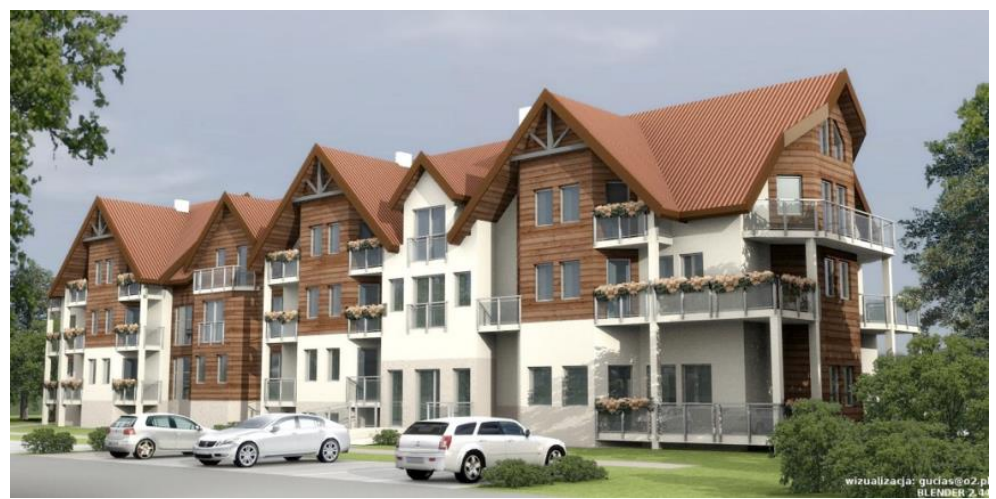

Figure 6 : Rendu final du même projet

Source : K. Nowacki

Autre outil, plus ergonomique que Blender, Google Sketchup ${ }^{42}$ est un logiciel de modélisation 3D essentiel en cartographie et en architecture. Par rapport aux autres logiciels $3 \mathrm{D}$, Sketchup ${ }^{43}$ fonctionne selon les principes de l'inférence : il émet des propositions en fonction de ce que l'utilisateur réalise. De plus, à la différence des autres logiciels de modélisation 3D, Sketchup permet de modéliser en temps réel. L'usager travaille directement sur le volume en extrudant ${ }^{44}$ ses faces, ajoutant une forme, tant et si bien qu'il devient facile de réaliser ses premiers bâtiments. Le rendu sous Sketchup permet d'obtenir

${ }^{42}$ Le logiciel Sketchup a été développé par la société @ Last Software, rachetée en mars 2006, par la société Google qui souhaitait un logiciel de modélisation d'objets en 3D, accessible à tous, pour Google Earth.

${ }^{43}$ Il existe deux versions de Sketchup : Google Sketchup qui est gratuite et Sketchup Pro, payante. La version gratuite possède toutes les fonctionnalités de base pour la modélisation et le rendu. Mais seule la version Pro dispose du module d'animation et présente l'avantage de pouvoir exporter dans tous les formats de dessin 3D usuels.

${ }^{44}$ En dessin vectoriel, extruder consiste à créer une forme tridimensionnelle à partir d'un objet plan. 
un croquis esquissé, tel un dessin technique avec les ombres portées et les textures photographiques. Voici quelques exemples :

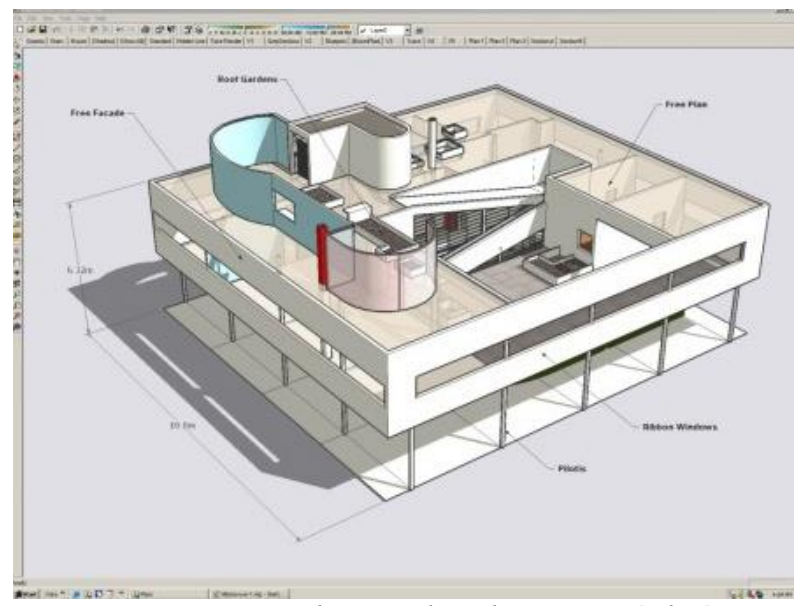

Figure 7 : Dessin technique d'un bâtiment réalisé avec Sketchup

Source : ziogeek

L'utilisation de «Google Sketchup » présente l'avantage. De pouvoir intégrer les rendus sur «GoogleEarth». Il existe un module qui permet de télécharger la dalle de Google Earth correspondant à la zone choisie et d'extruder les bâtiments directement au-dessus de l'orthophotographie. Les objets sont donc précisément géoréférencés, ce qui s'avère très utile pour l'insertion dans le MNT.

Ainsi, en s'appuyant sur les technologies du Web 2.0, tous ces logiciels libres ont pu être proposés en fonction des besoins des acteurs, qu'ils soient néophytes ou confirmés face à l'outil informatique, en vue d'une bonne gouvernance.

\section{En conclusion : les conditions pour une bonne gouvernance}

Pour répondre aux hypothèses de recherche, l'analyse des entretiens met en évidence que l'échelon local est perçu comme le plus pertinent pour la mise en place d'un projet d'aménagement du territoire répondant aux attentes et demandes des usagers. Toutefois, si à un échelon national l'aménagement rend difficile l'implication du citoyen, à un niveau trop localisé il ne concerne qu'une partie de la population. En cela, l'échelle du pays et du bassin de vie, dont l'outil de planification est le SCOT, assure un aménagement cohérent avec les besoins des usagers au sein d'un territoire. Cette échelle locale permet aux habitants de s'impliquer tout en mesurant les effets dans leur vie quotidienne.

La participation et l'intégration des usagers dans les différentes étapes du projet d'aménagement sont un des facteurs clé dans son acceptation et son appropriation. Imposer un projet sans aucune concertation auprès des habitants et usagers, l'expose inévitablement à des incompréhensions, des refus voire des conflits envers les décideurs. La participation collective étant un des piliers de la gouvernance, en impliquant davantage le citoyens, cela permet, d'une part, d'adapter la stratégie au plus exact des besoins de celui-ci, d'autre part, de réinstaurer le dialogue entre les élus et les administrés. 
Les applications du Web 2.0 sont devenues des outils essentiels à la gestion des territoires et fournissent un excellent support à la gouvernance. Les outils du Web 2.0 ont fait émerger des services et pratiques qui ont réellement permis de tisser des liens entre l'ensemble des acteurs au sein de leur territoire. Il est donc utile de mettre en place des supports innovants basés sur une plateforme d'échanges et de partage de données cartographiques en ligne. Les initiateurs d'un projet doivent pouvoir développer et utiliser cette plateforme afin d'y intégrer les usagers et permettre le partage et la gestion par la mise en réseau des acteurs. En Europe, des villes comme Rennes, Londres ont fait le choix de rendre accessibles leurs données de service public (cadastre, réseaux, transports...). La communauté peut donc avoir accès librement à ces données et de là émergent des idées, des savoir-faire, des compétences techniques, qui couplés avec la connaissance réelle des problèmes de terrain génèrent spontanément les outils permettant d'y remédier. Telle est l'innovation et la gouvernance des territoires à l'ère du Web 2.0.

L'innovation territoriale, avant d'être un concept économique et technologique, est un concept social. De nouveaux usages et de nouvelles pratiques, permettant de fédérer les habitants d'un territoire, sont rendus possibles par l'innovation sociale pour recréer du lien et du savoir vivre ensemble. Le lien social crée naturellement des dynamiques nouvelles qui vont alors générer une innovation bénéfique à l'économie d'un territoire. En cela, l'approche Living Lab constitue un formidable outil de gouvernance et d'innovation particulièrement adaptée à l'aménagement du territoire et au développement durable. La particularité d'un Living Lab est de placer l'usager au centre de la démarche, tout en favorisant la mise en réseau des acteurs, la participation collective, l'utilisation des outils informatiques et la production d'innovation sociale appliquée au territoire (Tchékémian, 2013).

\section{Références bibliographiques}

1. Boussard M., 2011. "Le Web 2.0 », LinkedIn, Slideshares.net, [URL : http://fr.slideshare.net/mickaelboussard29/le-web-20-7108271], consulté le 22 juillet 2016.

2. Brunet R., Ferras R, Théry H., 2005. Les mots de la géographie, dictionnaire critique, 480481,Ed. Reclus - La Documentation Française, nouvelle édition, Paris.

3. Calame P., 2008. "Les acteurs non étatiques et la gouvernance mondiale », FnGM, 2 juin 2008 [URL : http://www.world-governance.org/spip.php?rubrique37\&lang=fr], consulté le 2011 juin 20.

4. Casagrande L., 2011. Acteurs et gouvernance, innovation territoriale et aménagement durable : introduction à la démarche de Living Lab. Etude comparative de la gouvernance dans deux projets d'Ecoquartier, Tchékémian A. (dir.), mémoire de Master 2 Recherche, Université de Lorraine.

5. Charlot-Valdieu C., Outrequin P., 2009. L'urbanisme durable : Concevoir un écoquartier, Le Moniteur Editions.

6. Commission des Communautés Européennes, 2001. Gouvernance Européenne. Un livre blanc, Bruxelles, le 25 juillet 2001.

7. Duperrin B., 2008. "Le 2.0 vers une évolution systémique plus réaliste », Bloc-notes Des Hommes - Des entreprises - Des technologies, octobre 2008 [URL : http://www.duperrin.com/2008/10/02/le-20-vers-une-evolution-systemique-plus-realiste/], consulté le 13 juillet 2016 .

8. Eychenne F., 2009. La Ville 2.0, complexe et familière, FYP éditions. 
9. Farinos D. J., 2009, «Le défi, le besoin et le mythe de la participation à la planification du développement territorial durable : à la recherche d'une gouvernance territoriale efficace », 89, L'Information géographique, vol. 73, no2.

10.Fayon D., 2010. Web 2.0 et au-delà. Nouveaux internautes : du surfeur à l'acteur, 2ème éd., Economica.

11. Gaudin J-P., 2013. La démocratie participative, Ed. Armand Collin, 2ème édition, Coll. 128

12. Gerbaux F., Giraut F., 2000. "L'innovation territoriale. Références, formes et enjeux », Revue de Géographie Alpine, vol. 88, $\mathrm{n}^{\circ} 1$, 13-15, [URL : http://www.persee.fr/doc/rga_00351121_2000_num_88_1_2975], consulté le 30 juillet 2016.

13. Giraut F., 2009. "Innovation et territoires : les effets contradictoires de la marginalité », Revue de géographie alpine, 26 mai 2009, $\mathrm{n}^{\circ} 97-1$, mis en ligne le 25 mars 2010 [URL : http://rga.revues.org/783], consulté le 30 juillet 2016.

14. Kaplan D., Marcou T., 2009. La ville 2.0, plateforme d'innovation ouverte, Ed. FYP, Limoges.

15.Lacour C., Delamarre A., 2010, 40 ans d'aménagement du territoire, 6ème éd. actualisée, Coll. Territoires en mouvement, Ed. La Documentation Française, DATAR, Paris.

16.Lefèvre P., Sabard M., 2009, Les éco-quartiers : l'avenir de la ville durable, Ed. Apogée, Rennes.

17.Lévy J., 2011. Villes 2.0 - La recherche urbaine à l'heure de la ville 2.0, Ed. FYP, juin 2011, p. 46, mis en ligne le ler novembre 2011, [URL : http://doc.openfing.org/Villes2/Recherche_urbaine_villes2.pdf], consulté le 22 août 2011.

18.Lixon M., 2012, "Qu'est-ce qu'un Living Lab », France Silver éco, mis en ligne le 13 février 2012 [URL : http://www.cnr-sante.fr/2012/02/qu-est-ce-qu-un-living-lab/], consulté le 14 juillet 2016.

19. Musso P., Crozet Y., Joignaux G., 2002, Le territoire aménagé par les réseaux. Energie, transports et télécommunications, Ed. de l'Aube.

20. Renauld V., 2012. "Les écoquartiers à l'épreuve des usages ", L'implication des habitants dans les écoquartiers en France : quelles pratiques, quelles perspectives ?, Journée d'études, 18 septembre 2012, Lab'Urba-LET, ENSAPVS, pré-actes, pp. 108-122.

21.Tchékémian A., Richard G., 2013. «Innovation et gouvernance. La mobilisation des compétences et des ressources territoriales à travers le projet Living Lab "Innovation Santé Urbaine" à Nancy », In Chabault D., Hulin A., Leroy D., Soparnot R., La gestion des ressources humaines au service des réseaux d'innovation, chapitre 10, pp. 189-215, Ed. L'Harmattan, Coll. Points De Vue, Paris.

22. Vergnet-Covo M., 2009. La boîte à outils de la ville durable, Ed. Territorial, Coll. Les Classeurs, Voirons.

23. Wackermann G., 2005. Ville et environnement, Ed. Ellipses Marketing.

24.Wackermann G., 2008. France aménager les territoires, Ed. Ellipses, Coll. CAPES/Agrégation. 
